\title{
TRES TASAS MÁXIMAS DE GANANCIA EN SRAFFA
}

\author{
Antonio Mora Plaza
}

Economista, Madrid

http://dx.doi.org/10.5209/rev_NOMA.2012.v36.n4.42305

\begin{abstract}
Resumen.- En Producción de mercancías por medio de mercancías hay tres tipos de tasas máximas. Esta consideración puede ser considerada una sorpresa, porque Sraffa trabaja aparentemente con dos tasas máximas. Estas tres tasas son las siguientes: la de la razónpatrón, la de la tasa máxima calculada haciendo cero la tasa de salarios y la tasa que surge de tomar el mínimo de los excedentes (relativos) en porcentaje sobre las distintas mercancías. Este artículo trata de las relaciones entre estas tres tasas.
\end{abstract}

Palabras clave.- Sraffa, tipo, máximo, mínimo, excedente

\section{Three maximum rates in Sraffa}

Abstract.- There is in Production of commodities by means of commodities three kind of rates but not two only. This question may be considered a surprise because Sraffa also work with only two maximum rates. This topic never had been discovered to my knowledge. These rates are as following: the standard rate, the maximun rate calculated making zero the wage rate and the maximum rate taking the minimum rate the surplus in $\%$ by every commodities. This article is about the relations among these kinds of rates.

Keyswords.- Sraffa, rate, maximum, minimum, surplus

JEL: B51

\section{Introducción}

Cualquiera que haya leído la obra de Sraffa Producción de mercancías por medio de mercancías le resultará sorprendente el título que encabeza este artículo. Los más avezados en la obra del turinés entenderán que una de las tasas máximas sea la razón-patrón $\boldsymbol{R}$ en la producción simple porque en ésta, tal como la caracteriza Sraffa, la tasa máxima de ganancia coincide con la razón-patrón. Entonces, ¿cuál es la tercera tasa máxima que emplea, utiliza o se deduce de la obra de Sraffa? Lo más sorprendente es que el propio Sraffa no reconocería estas tres tasas porque él cometió el error de identificar una de ellas con una de las otras dos. Estas tres máximas de ganancia según su método de cálculo son: la de la razón-patrón en la producción simple, la de la tasa máxima de ganancia (tanto en la producción simple como en la producción conjunta) y la surgida del recuento de los excedentes relativos ${ }^{1}$. Esta es la

\footnotetext{
${ }^{1}$ Ambos términos -excedente relativo o su equivalente en inglés- no está en Sraffa, pero sí su concepto de forma inequívoca.
} 
tercera tasa en cuestión y que veremos que es ¡sorprendentemente! distinta de las otras dos. Veamos una por una.

\section{Razón patrón (R)}

Es ya muy conocida y es el icono de la producción simple junto con la mercancía-patrón. Surge como una propiedad de esta mercancía virtual que inventa Sraffa. Define ésta como aquella "en que las diversas mercancías están producidas en las mismas proporciones en que entran en los medios de producción totales"2. Dicho de otra forma, que el excedente relativo de todas las mercancías sea igual entre sí. Sraffa se refiere al conjunto de la economía y no a los medios empresariales o tecnológicos que tienen como causa (medios) el producto final. Un ejemplo: en una economía que se empleara 100 toneladas de acero procedentes del total de sectores o procesos y que se produjeran 120 toneladas también de acero, su excedente relativo sería del $20 \%$, independientemente de que la causa de producir las 120 toneladas no sean las 100 , sino el conjunto de medios empleados distintos del acero (aunque se empleara una pequeña parte del mismo acero también porque los componentes, por ejemplo, de los altos hornos también tuvieran acero o por el total de acero empleado indirectamente) ${ }^{3}$. Es una cesta virtual porque sólo una casualidad imposible de considerar sería que tal circunstancia se diera en el mundo real. Pero que sea virtual no quiere decir que no surta efecto en el mundo de la teoría económica abierta por Sraffa. Su principal virtud: que, a la postre, le permite al italiano establecer una relación entre salarios y ganancias que no depende de las variaciones de los precios. Con ello el sueño de Ricardo se ve cumplido a los 150 años de su muerte. La razón-patrón $\boldsymbol{R}$ se define a partir de las 4 ecuaciones siguientes:

$$
\begin{aligned}
& P Y=(1+g) P X+w L \\
& P Y=\left(1+g_{m}\right) P X \\
& P Y I-P X I=1 \\
& L I=1
\end{aligned}
$$

Y sin embargo esta razón-patrón $\boldsymbol{R}$ ¡no está... aún! en ninguna de este sistema de ecuaciones, en las que $\boldsymbol{Y}$ es la matriz ¡diagonal! $\boldsymbol{n} \times \boldsymbol{n}$ de $\boldsymbol{n}$ productos finales, $\boldsymbol{P}$ el vector de precios $\mathbf{1} \times \boldsymbol{n}, \boldsymbol{g}$ la tasa de ganancia, $\boldsymbol{w}$ la tasa de salarios, $\boldsymbol{L}$ el

\footnotetext{
${ }^{2}$ Pág. 40 de $P M P M$.

${ }^{3}$ Esta es la razón por la que Sraffa señala con cierta sorna en el prefacio de que el no hace ningún supuesto de rendimientos constantes, pero que "si encuentra útil tal supuesto no hay inconveniente en que el lector lo adopte como una hipótesis temporal de trabajo" (Pág. 12 de $P M P M$, edit. Oikos-Tau). Los rendimientos (marshallianos) constantes o no se refieren a una empresa y siempre que haya una relación causa-efecto, cosa imposible en el modelo de Sraffa, lo que convierte en anécdota lo de la constancia. De ahí el malévolo comentario de Sraffa en este punto. Keynes, cuando leyó el manuscrito supuestamente aún inacabado, le comentó que hacía el supuesto de rendimientos constantes.
} 
vector de inputs de trabajo $\mathbf{1} \times \boldsymbol{n}, \boldsymbol{g}_{\mathrm{m}}$ es la tasa máxima de ganancia cuando se hace cero la tasa de salarios $\boldsymbol{w}$ en (1) y $\boldsymbol{X}$ la matriz no diagonal $\boldsymbol{n} \times \boldsymbol{n}$ de medios de producción. La razón de ello es que en la ecuación (2) surge sólo de hacer cero la tasa de salario $\boldsymbol{w}$ en (1). Sraffa creía que $\boldsymbol{g}_{\mathrm{m}}$ era la razón-patrón $\boldsymbol{R}$ y lo razona desde el punto de vista económico en el capítulo $\mathrm{V}$ de su obra que trata del "carácter único del sistema patrón". Dice Sraffa en ese capítulo que "puede demostrarse que el valor de $\boldsymbol{R}$ al que corresponden todos los precios positivos es el mínimo de todos los $\boldsymbol{k}$ posibles" ${ }^{4}$. Veremos que la frase de Sraffa es totalmente desafortunada porque es falsa, a pesar de lo cual, su obra avanza genialmente hasta el mismo apéndice $B$ de los productos que se autoreproducen. Nos quedamos de momento con esto y avanzamos por nuestra parte que la razón-patrón se obtiene del sistema de ecuaciones:

$$
\begin{aligned}
& u Y Q=X Q \\
& L Q=1
\end{aligned}
$$

En (5), $\boldsymbol{u}$ es el autovalor máximo de la matriz $\boldsymbol{A}=\boldsymbol{Y}^{-1} \boldsymbol{X}$ y $\boldsymbol{Q}$ es el vector columna $n \times \mathbf{1}$ de multiplicadores que sirven para construir la mercancía-patrón que es el sistema (5) de $\boldsymbol{n}$ ecuaciones. Si ahora pre-multiplicamos por los precios $\boldsymbol{P}$ la (5) y pos-multiplicamos la (2) por los multiplicadores $Q$ queda:

$$
u P(Y Q)=P(X Q)
$$

\footnotetext{
${ }^{4}$ Pág. 51 de PMPM. Tal es así que Sraffa, según criterio de Bertram Schefold nunca conoció el teorema de Perron-Frobenius. Dice Schefold en Joint Production: Triumph of Economic over Mathematical logic?: "Perron published his theorem in 1909, Frobenius his in 1912 in Germany. Several others proofs were published around 1950, but neither Sraffa nor his mathematicians took notice of them", pag. 175, Routledge, 2009. Sin embargo Kurz y Salvadori recogen la duda en Sraffa and the mathematicians: Frank Ramsey and Alister Watson cuando dicen que "As regards the second remark, as reported by Sraffa, we do not know, of course, what was at the back of Ramsey's mind. However, had the starting point of his remark been the PerronFrobenius Theorem, then things would have been crystal clear. Yet in this case he could have been expected to draw Sraffa's attention to the existence of this theorem, which is a most powerful tool to solve the kind of problems Sraffa was interested in. There is no evidence to this effect; on the contrary, Sraffa's papers would seem to imply that none of his mathematical friends referred him to this theorem". Parece pues que, a pesar de los esfuerzos de sus amigos -especialmente del sufrido Ramsey- y a pesar de que el teorema dejaba claro el problema ("cristal clear"), Sraffa o no lo tuvo en cuenta o no lo llegó a conocer realmente. La ventaja es que tal desconocimiento le obligó a un esfuerzo explicativo que, a pesar de sus errores formales, dio como resultado un libro (Producción de...) de economía y no sólo un mero modelo matemático a la manera de Von Neumann. No hay bien que por mal no venga.

${ }^{5}$ La ecuación $L Q=1$ es una genialidad más de Sraffa porque permite añadir una ecuación al sistema de (5) cuando lo necesitaba isin añadir ninguna nueva variable! En efecto, los inputs $\mathbf{I}_{\mathrm{i}}$ de trabajo se consideran constantes y el vector de multiplicadores $\mathbf{Q}$ ya han aparecido en (5). La necesidad de una nueva ecuación se derivaba de que en (5) hay $\mathbf{n}$ ecuaciones, peros $\mathbf{n + 1}$ variables: el autovalor $\mathbf{u}$ y los $\mathbf{n}$ multiplicadores $\mathbf{q}_{\mathrm{j}}$. Al añadir la ecuación (5bis) a las $\mathbf{n}$ ecuaciones de (5), el número de ecuaciones se iguala con el de incógnitas y el sistema tiene -0 puede tener- solución. Además, como subproducto de este proceder, se incorporan los inputs de trabajo que, de lo contrario, no hubieran jugado ningún papel, lo cual dejaría al sistema patrón y a la propia mercancía patrón (5) bajo sospecha.
} 


$$
(P Y) Q=\left(1+g_{m}\right)(P X) Q
$$

$Y$ los sistemas de ecuaciones (6) y (7) son el mismo si hacemos que $\boldsymbol{u}=\mathbf{1} /\left(\mathbf{1}+\boldsymbol{g}_{\mathrm{m}}\right)$, o de otra manera, $\boldsymbol{g}_{\mathrm{m}}=(\mathbf{1}-\boldsymbol{u}) / \boldsymbol{u}$. Sraffa no nos dice nada de esto porque en ningún momento emplea -aunque lo conociera porque tuvo tres magníficos matemáticos que le asesoraron ${ }^{6}$ - el teorema de Perron-Frobenius. Este teorema nos dice que, dada una matriz $\boldsymbol{A}$ (en este caso concreto, $\boldsymbol{A}=\boldsymbol{Y}^{-1} \boldsymbol{X}$ ) cuadrada, positiva e irreducible (versión fuerte del teorema) existen dos autovectores de $\boldsymbol{A}$-una por la izquierda y otro por la derecha- y un autovalor, que es el máximo de los autovalores tomados en su valor absoluto y que es el único de los autovalores, que hace que esos dos autovectores (en este caso, los precios y/o los multiplicadores) sean estrictamente positivos. Sraffa no sólo no habla de todo esto sino que no lo emplea, y construye su razón-patrón como el menor de lo $\boldsymbol{k}$, es decir, el menor de los excedentes relativos posibles. Al sostener la igualdad $\boldsymbol{g}_{\mathrm{m}}=(\mathbf{1}-\boldsymbol{u}) / \mathbf{u}$ merced al teorema mencionado, llegamos a que $\boldsymbol{g}_{\mathrm{m}}=\boldsymbol{R}$. Y con ello estamos ya en la razón-patrón $\boldsymbol{R}$ como, además, tasa máxima de ganancia $\boldsymbol{g}_{\mathrm{m}}$. Sraffa plantó la semilla -que era lo importante-, aunque la plantara en lugar equivocado. Llegado a ese punto de igualdad entre excedente y tasa máxima, la razón-patrón deja de ser una variable monetaria para convertirse en una variable que depende sólo de los medios $\boldsymbol{X}$, de los productos finales $Y$ y de los inputs de trabajo $L$ (merced al numerario $L \mathbf{L}=\mathbf{1})^{7}$. La segunda consecuencia de que la tasa máxima de ganancia sea la razón-patrón en la producción simple es que ahora $\boldsymbol{R}$ no añade ninguna variable al sistema, cosa que ocurriría si ambas -tasa de ganancia máxima y razón-patrón- fueran distintas.

\section{Tasa de ganancia máxima $\left(\mathbf{g}_{\mathrm{m}}\right)$}

Esta tasa surge también de hacer cero la tasa de salarios en la ecuación de definición del sistema, tanto en la producción simple como conjunta. Este último sistema lo caracteriza Sraffa como aquel en el que "dos mercancías son producidas conjuntamente por una sola industria". a partir de él podemos decir que un sistema de producción conjunta es aquel en el que $\boldsymbol{n}$ industrias, sectores o procesos producen hasta $\boldsymbol{m} \times \boldsymbol{n}$ posibles productos finales. La ecuación que define el sistema de producción conjunta versión Sraffa- es similar a de la producción simple:

$$
P Y=w L+(1+g) P X
$$

pero con la notable diferencia de que, en este caso, la matriz $Y$ no es una matriz diagonal, sino una matriz con posibles $n \times n$ elementos positivos. Ello da

\footnotetext{
${ }^{6}$ Besicovitch, Ramsey y Watson. Es el propio Sraffa quien hace reconocimiento a estos matemáticos en su prólogo a su libro Producción de...

7 Ver apéndice.

${ }^{8}$ Pág. 67 de $P M P M$.
} 
lugar a una matriz de requerimientos ${ }^{9} \boldsymbol{A}=\boldsymbol{X} \boldsymbol{Y}^{-1}$ en la que ya no podemos asegurar que todos sus elementos $\boldsymbol{a}_{\mathrm{ij}}$ sean todos positivos, razón suficiente para que ya no se pueda aplicar el teorema de Perron-Frobenius, con lo cual no tenemos autovalor que de lugar a precios estrictamente positivos 0 , alternativamente, unos multiplicadores todos positivos. Sraffa no emplea estos argumentos al no considerar el teorema y recurre para ello al método del menor de los excedentes relativos. Es verdad que con ello llega -aunque no lo demuestra- también a un vector de precios estrictamente positivo, pero se equivoca al considerarlo como condición necesaria para obtener el vector positivo cuando en realidad, a lo sumo, es una condición suficiente. Por eso no es errónea lo que dice Sraffa en el comienzo del capítulo VIII: "tan pronto como consideramos en detalle la construcción de un sistema patrón con productos conjuntos, resulta obvio que puede que algunos de los multiplicadores tengan que ser negativos"10. De (8) -al igual que de (1)- se obtiene la ecuación de precios:

$$
P=w L Y^{-1}[I-(1+g) A]^{-1}
$$

donde, al estar en la producción conjunta, ya no podemos asegurar que todos los elementos de $A=X Y^{1}$ sean positivos; tampoco lo podemos decir de los $L Y^{1}$. El resultado es que ya no se puede garantizar que todos precios sean positivos en (9).

\section{Tasa de ganancia-recuento (k)}

Ya ha salido antes, pero la cuestión es que Sraffa nunca utilizó PerronFrobenius y creyó que su método de recuento de los excedentes relativos era equivalente al del teorema. $O$ al menos hay que suponer que conocía el teorema y no lo quiso utilizar para evitar que su obra se deslizara hacia un mero modelo matemático a la manera del de Von Neumann o los de equilibrio general a la manera de Walras. Para Sraffa la razón-patrón es igual al mínimo de los excedentes relativos de todas las mercancías ${ }^{11}$. Es decir:

$$
k=\text { menor } k_{i}=\frac{Y_{i}-\sum_{j=1}^{n} X_{i j}}{\sum_{j=1}^{n} X_{i j}} \forall i=1 \text { a } n
$$

\footnotetext{
${ }^{9}$ El autovalor del producto de dos matrices cuadradas tiene la propiedad conmutativa, es decir, autovalor de $\mathbf{Y}^{-1} \mathbf{X}=$ autovalor de $\mathbf{X} \mathbf{Y}^{-1}$, siempre, claro está, que ambas matrices sean no negativas, cuadradas e indescomponibles. En la producción simple se cumple la no negatividad (con más propiedad, la semipositividad) porque $Y$ es diagonal, con lo que la inversa también lo es.

${ }^{10}$ Pág. 71 de PMPM.

11 "Como consecuencia inmediata de lo anterior, puede demostrarse que el valor de $\mathrm{R}$ al que corresponden todos los precios positivos es el mínimo de todos los k posibles" (PMPM, pág, 51)
} 
Sraffa razona que si la tasa de ganancia es menor o a lo más igual que esta tasa basada en el recuento de los excedentes relativos, los precios no podrán ser negativos en la producción simple. Para la producción conjunta ya queda dicho que Sraffa, con acierto, razona que ya no se puede asegurar que todos los precios sean positivos por los efectos indirectos de las diferentes proporcionalidades de los medios en relación a los productos todos aquellos que intervienen en la producción, sea de forma directa y/o indirecta. Veremos que esto es sólo una verdad a medias. Resulta sorprendente que ningún crítico o historiador de Sraffa se haya dado cuenta de este hecho. El caso es que no afecta a las conclusiones económicas de Sraffa en toda su obra, pero sí en la base formal de su sistema.

\section{Relación entre estas tres tasas}

La pregunta que surge llegado este punto es: ¿qué relación guardan estas tres tasas? ¿Pueden compararse o pertenecen a supuestos diferentes que no admiten comparación? Veamos un sistema de ecuaciones que nos van a permitir comparar la tasa de ganancia máxima y la razón-patrón. Damos el sistema de ecuaciones para posterior explicación:

$$
\begin{aligned}
& \underset{1 \times m}{P_{N}} Y_{N}+\underset{1 x n}{P} \underset{n x n}{Y}=w \underset{1 x n}{L}+(1+g) \underset{1 \times n \text { nxn }}{P} \\
& P_{N} Y_{N}+P Y=\left(1+g_{m}\right) P X \\
& P Y=(1+R) P X \\
& P Y I-P X I=1 \\
& L I=1
\end{aligned}
$$

En este sistema hay bienes no-básicos representados por la matriz $Y_{\mathrm{N}}$ de dimensiones $\boldsymbol{m} \times \boldsymbol{n}$ y sus respectivos precios $\boldsymbol{p}_{\mathrm{m}}$ representados por el vector $\boldsymbol{P}_{\mathrm{N}}$ de dimensión $\mathbf{1 x m}$. El resto de las ecuaciones ya han sido comentadas, y especialmente el hecho de que, a pesar de estar en la producción conjunta por existir bienes no-básicos "desgajados" de los básicos, la matriz $Y$ de productos finales es, en este caso, una matriz diagonal ${ }^{12}$ (como en la reproducción simple). Es decir, el sistema de ecuaciones anterior caracteriza una economía híbrida entre la producción simple ( $Y$ diagonal) y conjunta (existencia de bienes no básicos $Y_{\mathrm{N}}$ ). De las ecuaciones (14) y (15) obtenemos:

\footnotetext{
${ }^{12}$ Este supuestos es fundamental y único para poder encauzar en un mismo sistema de ecuaciones la razón-patrón y la tasa máxima de ganancia. Si Y no fuera diagonal como en la producción conjunta típicamente esrafiana, entonces ya no tendríamos mercancía-patrón; aún así se puede concebir una forma de mercancía-patrón, pero con el inconveniente señalado por el propio Sraffa de que algunos de los multiplicadores fueran negativos.
} 


$$
P_{N} Y_{N} I=\left(g_{m}-R\right) P X I
$$

Y en (18) se aprecia claramente que si se quiere que el valor del conjunto de los bienes de consumo (no-básicos, dicho en términos esrafianos) $\boldsymbol{P}_{N} \boldsymbol{Y}_{N} \mathbf{l}$ sea mayor que cero, ha de ocurrir que la tasa de ganancia $\boldsymbol{g}_{\mathrm{m}}$ sea mayor que la razón-patrón $\boldsymbol{R}$, es decir, que:

$$
\text { si } P_{N} Y_{N} I \geq 0 \Rightarrow g_{m} \geq R
$$

En (19) vemos la ventaja de la razón-patrón $\boldsymbol{R}$ sobre la tasa máxima de ganancia $\boldsymbol{g}_{\mathrm{m}}$, porque, sin necesidad de que el sistema haga desaparecer los salarios (criterio de la tasa máxima), se puede obtener un vector de precios positivos dado que eso nos lo asegura la razón-patrón, que es a la vez una medida del excedente y la tasa máxima de ganancia. En el caso de que fueran cero los productos no básicos habríamos llegado al paraíso esrafiano de la producción simple porque, recordémoslo, en este modelo propuesto, la matriz de productos finales no es diagonal, sino que todos sus elementos tienen valor mayor o igual a cero.

Ahora vayamos a la tasa de ganancia basada en el recuento de los diferentes excedentes relativos y que hemos visto en (12):

$$
k=\text { el menor de todos los } k_{i}=\frac{Y_{i}-\sum_{j=1}^{n} X_{i j}}{\sum_{j=1}^{n} X_{i j}} \forall i=1 \text { a n }
$$

La ecuación (20) en términos matriciales puede expresarse como:

$$
P Y=P(I+K) X
$$

siendo $\boldsymbol{K}$ la matriz diagonal formado por los $\boldsymbol{n}$ excedentes relativos $\boldsymbol{k}_{\mathrm{ii}}$ en principio todos distintos. Ahora, si comparamos (21) con (15) se obtiene:

$$
P Y=P(I+K) X=(1+R) X
$$


Y si en (22), no tomamos los $\boldsymbol{n}$ diferentes excedentes relativos $\boldsymbol{k}_{\mathrm{ii}}$, sino el menor de todos ellos, es decir $\boldsymbol{k}$, de acuerdo con (20), lo que tenemos es:

$$
P(I+k) X \leq(1+R) P X \Rightarrow k \leq R
$$

Y el resultado de unir (23) con (19) es la notable:

$$
k \leq R \leq g_{m}
$$

que es la relación que queríamos obtener. Nos dice que la tasa de de ganancia máxima $\boldsymbol{g}_{\mathrm{m}}$ es mayor que la razón-patrón $\boldsymbol{R}$ y que esta lo es de la tasa-recuento $\boldsymbol{k}$ (Sraffa), aunque podría haber algún caso extremo en que fueran iguales. Esta relación (24) es válida para la producción simple; también lo es para el sistema híbrido de simple y conjunta que hemos fabricado y comentado; sólo lo es la relación entre el método del recuento $\boldsymbol{k}$ y el de la tasa máxima $\boldsymbol{g}_{\mathrm{m}}$ para la producción conjunta estricta porque, en este sistema de producción, no se puede construir la razón-patrón $\boldsymbol{R}$ tal como se hace en la producción simple. Ni Sraffa ni en este artículo se ha demostrado que el método de recuento de Sraffa resultó válido como condición suficiente; sí hemos demostrado, en cambio, que no es condición o requisito necesario en la producción simple y en la híbrida, porque esta condición sólo la cumple la razón-patrón por mor de Perron-Frobenius. Sólo la razón-patrón $\boldsymbol{R}$ nos asegura precios estrictamente positivos sin partir del mínimo de los excedentes relativos y sin llegar al máximo de la tasa de ganancia. Cuando, por las características del sistema que hemos definido con nuestros conceptos, reglas y ecuaciones, no podemos aplicar Perron-Frobenius, tenemos que recurrir a lo que decía Sraffa, es decir, a que serán los propios empresarios y/o actores de la escena económica los que, con su comportamiento económico, evitarán llegar a extremos como precios negativos o precios tendentes al infinito ${ }^{13}$ (las habas del apéndice $B$ del libro de Sraffa).

Un comentario aparte. Parecería por la discusión anterior y las variables implicadas que estamos muy alejado de la realidad y que hubiéramos sacrificado ésta en aras de lo explicativo porque: a) empleamos tasas unitarias para salarios y ganancias; b) porque la mercancía-patrón es una construcción virtual. Pues nada de eso. La realidad está inserta en el modelo porque, sin dejar de serlo, podemos hacer las cosas siguientes: a) podemos arribar a las tasas unitarias desde ecuaciones de tipo $\boldsymbol{w} L \mathbf{L}=\boldsymbol{L} \boldsymbol{W I}$ y $\boldsymbol{w} \mathbf{L} \mathbf{I}(\mathbf{1}+\boldsymbol{g}) \boldsymbol{P X |}=\boldsymbol{L} \boldsymbol{W I}+\boldsymbol{P} X(\mathbf{I}+\boldsymbol{G}) \mathbf{I}$, con $\mathbf{G}$ como matriz diagonal $\boldsymbol{n} \times \boldsymbol{n}$ de $\boldsymbol{n}$ tasas de

\footnotetext{
${ }^{13}$ Lo emplea Sraffa cuando quiere deducir los posibles efectos de los precios ante un aumento de lo salarios (pág. 87 de PMPM), pero el criterio puede extenderse a cualquier solución matemática del sistema que no tuviera sentido económico.
} 
ganancia en lugar del escalar $\boldsymbol{g}$; b) podemos partir de salarios pre-factum en lugar de los post-factum esrafianos, es decir, incluir en las tasas de ganancia todos los costes, es decir, hacer que $\boldsymbol{P Y}=(\boldsymbol{L} \boldsymbol{W}+\boldsymbol{P X})(\mathbf{I}+\boldsymbol{G}) ; \mathrm{c})$ porque podemos hacer que $\boldsymbol{Y}_{\mathrm{N}}$ no sea cuadrada, con $\boldsymbol{m} \times \boldsymbol{n}$ mercancías distintas de $\boldsymbol{n}$ sectores (o procesos) sin que varíen las conclusiones; d) porque la matriz $\boldsymbol{W}$ tampoco tiene que ser diagonal (incluso tampoco cuadrada, puesto que no requerimos su inversa); e) porque podemos tomar $\boldsymbol{n}$ tasas máximas de ganancia $\boldsymbol{G}_{\mathrm{m}}$ mediante $\left(\mathbf{1}+\boldsymbol{g}_{\mathrm{m}}\right) \boldsymbol{P} \boldsymbol{X I}=\boldsymbol{P} \boldsymbol{X}\left(\mathbf{I}+\boldsymbol{G}_{\mathrm{m}}\right) \mathbf{I}$; f) porque $\boldsymbol{g}_{\mathrm{m}}$ es linealmente dependiente de $\boldsymbol{X}^{-1}(\boldsymbol{Y}-\boldsymbol{X})^{-1}$, es decir, de los valores físicos de medios, productos e inputs de trabajo (esto último merced al numerario ${ }^{14} \mathbf{L I}=\mathbf{1}$ ).

Resumiendo, Sraffa, en su afán de eludir al menos en la fase expositiva de su modelo las matemáticas, cometió al menos dos errores: 1) creyó que la tasa máxima de ganancia era conceptualmente igual que la razón-patrón en la producción simple cuando sólo lo era cuantitativamente; 2) creyó que el método del recuento de los excedentes relativos podía llevarle a la tasa máxima de ganancia en la producción conjunta. Ambas cosas son falsas como se ha demostrado. Eso, no obstante, no aminora el hecho de que Producción de mercancías por medio de mercancías sea uno de los 3 o 4 textos más importantes de la historia del análisis económico (en lo que atañe a los fundamentos, en mi opinión el que más y casi único).

\section{Apéndice I}

Una forma de abordar la estimación de las tasas máximas de ganancia $\boldsymbol{G}_{\mathrm{m}}$ es como sigue. Por un lado tenemos la ecuación de precios derivada de la definición del sistema:

$$
P=L W\left(I_{d}+G\right)[Y-X(I+G)]^{-1}
$$

Por otro, tenemos la ecuación resultante de dos: la de definición del sistema $\boldsymbol{P Y}=(\boldsymbol{L} \boldsymbol{W}+\boldsymbol{P X})(\mathbf{I}+\boldsymbol{G})$ y la de hacer cero la tasa de salarios que es $\boldsymbol{P} \boldsymbol{Y}=\boldsymbol{P X}\left(\mathbf{I}+\boldsymbol{G}_{\mathrm{m}}\right)$. De entre ambas sale:

$$
P=L W\left(I_{d}+G\right)\left[\left(G_{m}-G\right)^{-1} X^{-1}\right]
$$

A simple vista se puede observar que se puede conjeturar una relación lineal entre la resultante de la matriz entre corchetes de (1) y la de (2), y por ello se puede establecer la siguiente relación:

\footnotetext{
${ }^{14}$ Tomar un numerario es una manera de decir que una ecuación -todas si hay más de unahan sido divididas por la expresión del numerario. En este caso, que hemos divido todas las ecuaciones entre LI. También hemos tomado otros numerarios compatibles con el anterior puesto que en ningún caso se repiten las variables.
} 
(3)

$$
\left[Y-X\left(I_{d}+G\right)\right]^{-1}=\left[\left(G_{m}-G\right)^{-1} X^{-1}\right] F
$$

siendo $\boldsymbol{F}$ una matriz diagonal que podemos calcular porque tenemos $\boldsymbol{n}$ ecuaciones y $\boldsymbol{n}$ incógnitas. Si ahora hacemos igual a cero las tasas de ganancia, la nueva relación de (3) queda:

$$
[Y-X]^{-1}=G_{m}^{-1} X^{-1} F
$$

Y pre-multiplicando (4) por $\boldsymbol{G}_{\mathrm{m}}$ y post-multiplicando por $(\boldsymbol{Y}-\boldsymbol{X})$ sale:

(5)

$$
G_{m}=X^{-1} F(Y-X)
$$

Y conocido $\boldsymbol{F}$ se puede encontrar otra matriz $\boldsymbol{H}=f(\boldsymbol{F})$ tal que:

(6)

$$
G_{m}=H X^{-1}(Y-X)
$$

La ecuación (6) nos dice que las tasas máximas de ganancia son proporcionales a los excedentes relativos $\left(X^{-1}(Y-X)\right)$

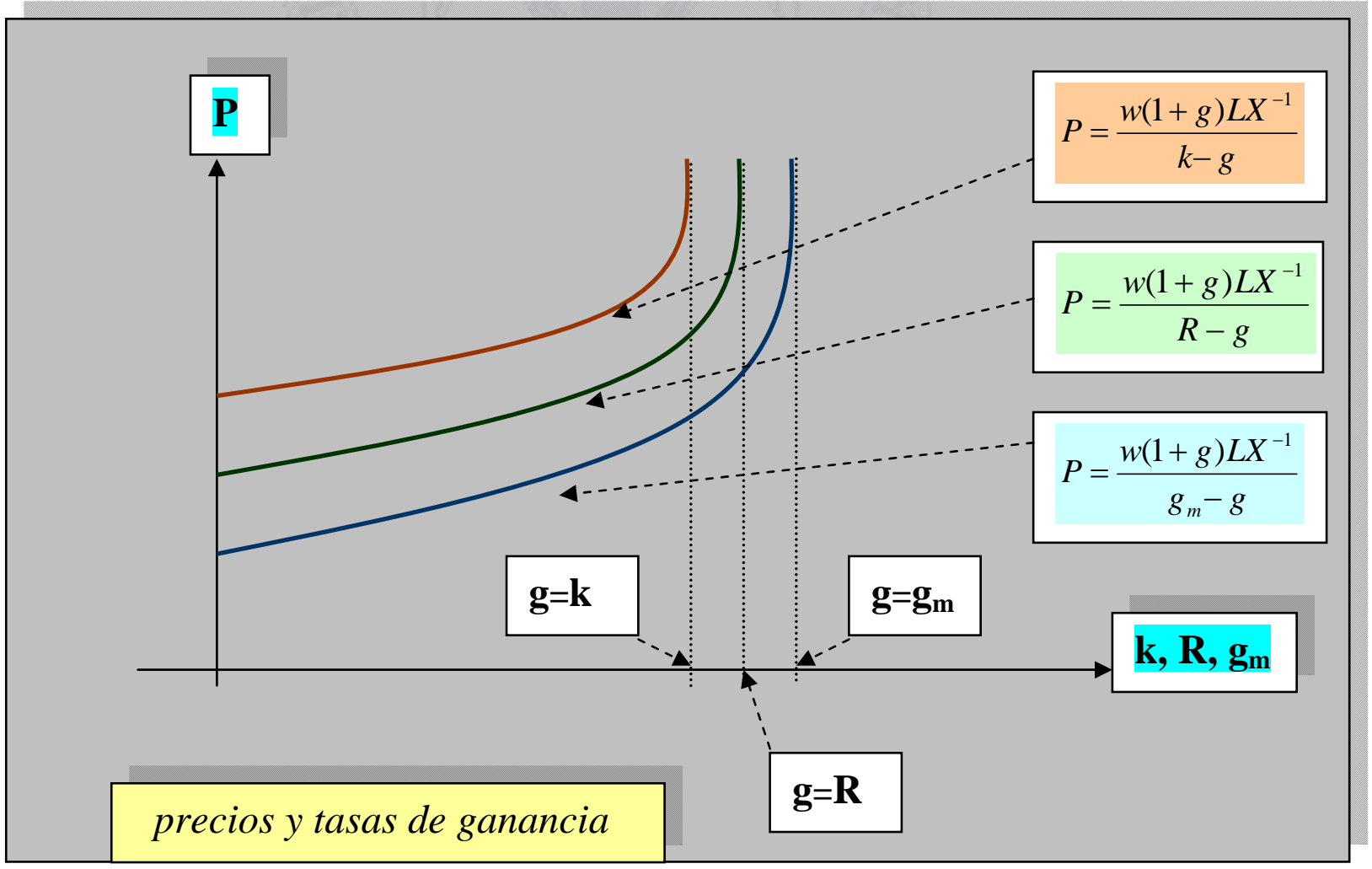




\section{Apéndice II}

Esta confusión del aspecto cualitativo y cuantitativo entre tasa máxima de ganancia y razón-patrón que se desprende de la lectura de Producción de mercancías por medio de mercancías proviene de que en la producción simple y para la mercancía-patrón, ambas son cuantitativamente iguales. En este apéndice vamos a demostrarlo. Sea una ecuación de definición del sistema, pero de la que partimos con los valores $Y$ de productos finales y de medios de producción $\boldsymbol{X}$ obtenidos de la mercancía-patrón:

$$
P_{q} Y Q=w L Q+(1+g) P_{q} X Q
$$

Puesto que estamos en la producción simple $\boldsymbol{Y}$ es una matriz diagonal $\boldsymbol{n} \times \boldsymbol{n}$. La particularidad ahora de este sistema es que $\boldsymbol{Q}$ es una matriz $\boldsymbol{n} \times \boldsymbol{n}$ diagonal, donde los elementos de la diagonal principal son los multiplicadores obtenidos de la mercancía-patrón definida como siempre:

$$
u Y Q I=X Q I
$$

donde I es el vector de unos $n \times \mathbf{1}$. Los precios los hemos llamado $\boldsymbol{P}_{\mathrm{q}}$ porque no necesariamente han de ser los mismos que los surgidos de la ecuación esrafiana de definición del sistema $\boldsymbol{P Y}=(\mathbf{1}+\boldsymbol{g})(\boldsymbol{w} \boldsymbol{L}+\boldsymbol{P X})$ dada la presencia de los multiplicadores $\boldsymbol{Q}$. Ello no importa, porque los precios van a desaparecer en lo que sigue, es decir, no van a jugar ningún papel en la demostración, lo cual significa que la demostración es válida para cualquier nivel de precios. El producto QI sería los multiplicadores habituales colocados en un vector vertical directamente. Si ahora hacemos cero la tasa de salarios tal y como hace Sraffa, sale que:

$$
P_{q} Y Q=\left(1+g_{m}\right) P_{q} X Q
$$

donde $\boldsymbol{g}_{\mathrm{m}}$ es la tasa máxima de ganancia. Hacemos ahora de forma análoga cero la tasa de ganancia $\boldsymbol{g}$ para conseguir la ecuación correspondiente a la tasa máxima de salarios:

$$
P_{q} Y Q=w_{m} L Q+P_{q} X Q
$$


De las ecuaciones (3) y (4) se obtiene la ecuación de precios dependiente de ambas tasas máximas:

$$
P_{q}=\frac{w_{m}}{g_{m}} \times L X^{-1}
$$

donde vemos que ha desaparecido, tanto de $\boldsymbol{L}$ como de $\boldsymbol{X}$, la matriz de multiplicadores $\boldsymbol{Q}$. Y si ahora sustituimos los precios de (5) en la ecuación (3), sale la ecuación de la tasa máxima de ganancia:

$$
g_{m}=\frac{L X^{-1}[Y-X] Q I}{L Q I}
$$

Esta ecuación puede ser escrita de la forma:

$$
g_{m}=\frac{L X^{-1} Y Q I}{L Q I}-1
$$

Vamos ahora a la ecuación de definición de la mercancía-patrón en (2) y la premultiplicamos por la inversa de $\boldsymbol{X}$. El resultado es:

$$
u X^{-1} Y Q I=Q I
$$

Ahora la pre-multiplicamos por el vector horizontal de inputs de trabajo $\boldsymbol{L}$ y pasamos el factor de escala $\boldsymbol{u}$ al otro lado de la igualdad:

$$
L X^{-1} Y Q I=\frac{1}{u} L Q I
$$

Y ya sólo queda sustituir $L X^{-1} Y Q I$ de (9) en (7) y sale:

$$
g_{m}=\frac{\frac{1}{u} L Q I}{L Q I}-1=\frac{1-u}{u}
$$


Pero en la producción simple la razón-patrón $\boldsymbol{R}$ es igual a (1-u)/u siempre que $\boldsymbol{u}$ sea el autovalor de mayor valor (absoluto) de la matriz $X Y^{-1}$, cuya resultante es no negativa porque $Y$ es diagonal y positiva. De ello se deduce que:

$$
g_{m}=\frac{1-u}{u}=R
$$

Y aquí acaba la demostración. En la producción conjunta esta igualdad no se puede mantener, aun cuando partiéramos de economía de mercancía-patrón construida sin Perron-Frobenius- porque ya no tenemos una matriz de productos finales $Y$ que sea diagonal.

\section{Apéndice III}

Cuando tuve redactado el artículo completo -junto con los dos apéndices anteriores- me di cuenta que Sraffa comete el error de manejar dos numerarios a la vez de forma indebida. No es que ello no esté permitido, pero tal como lo hace el economista italiano lleva a considerar que el valor del producto neto (PYI-PXI) y la suma de los inputs de trabajo (LI) tienen el mismo valor. Ello es sólo posible por casualidad. En efecto, Sraffa parte de las ecuaciones ya señaladas:

$$
\begin{aligned}
& P Y=(1+g) P X+w L \\
& P Y=\left(1+g_{w=0}\right) P X \\
& P Y I-P X I=1 \\
& L I=1
\end{aligned}
$$

Luego explicaremos porque llamamos $\boldsymbol{g}_{\mathrm{w}=0}$ a la tasa máxima de ganancia en lugar de la empleada en el texto del artículo $\boldsymbol{g}_{\mathrm{m}}$. Estos 4 sistemas de ecuaciones matriciales nos llevan a la expresión cuasi-esrafiana:

$$
g=(1-w) g_{w=0}
$$

Sraffa considera que la tasa máxima de ganancia definida en (2) es la razónpatrón $\boldsymbol{R}$, por lo que (5) se convierte en:

$$
g=(1-w) R
$$


Y ya tenemos el reparto del excedente $(\boldsymbol{R})$ entre ganancias $(\boldsymbol{g})$ y salarios $(\boldsymbol{w})$ ¡independiente de los precios $\boldsymbol{P}$ ! Pero tomar las ecuaciones (3) y (4) como numerarios supone considerar que se cumpla que:

$$
P Y I-P X I=L I
$$

lo cual sólo es posible por casualidad. Y dado que considerar como numerario una relación entre variables -o una variable- supone dividir el conjunto de ecuaciones de definición del sistema entre esa expresión, la expresión resultante de (1) con los numerarios (3) y (4) daría lugar a:

(8)

$$
\frac{1}{(P Y I-P X I) \times L I} \times P Y=(1+g) \frac{1}{(P Y I-P X I) \times L I} \times P X+\frac{1}{(P Y I-P X I) \times L I} \times w L
$$

Dicho de otra forma, al tomar simultáneamente los numerarios (3) y (4), Sraffa tomó en realidad como numerario $(\boldsymbol{P Y I}-\boldsymbol{P X I}) \mathbf{L I}$. Nadie hasta ahora que sepa ha advertido de tal error. ¿Qué hacer? Vamos a considerar que operamos bajo un solo numerario. El elegido será el producto neto, es decir, PYI-PXI. El sistema de ecuaciones ahora sería entonces:

$$
\begin{aligned}
& P Y=(1+g) P X+w L \\
& P Y=\left(1+g_{w=0}\right) P X \\
& P Y I-P X I=1
\end{aligned}
$$

Y de la resolución del sistema de ecuaciones anterior nos da:

$$
g=(1-w L I) g_{w=0}
$$

dado que ahora -a diferencia del modelo de Sraffa- $L \mathbf{I}$ no es un numerario y no se cumple que $L \mathbf{L I}=\mathbf{1}$.

El segundo aspecto que vamos a considerar es qué podemos entender como tasa máxima de ganancia fuera ya del sistema de Sraffa. Ya hemos demostrado -0 al menos intentado- que Sraffa trabaja con 3 tipos de tasas 
máximas, aunque el no fuera consciente de ello. A lo sumo lo fue de 2. Para calcular la tasa máxima de una variable en función de otra, el cálculo infinitesimal de Descartes, Newton $Y$ Leibniz ( $y$ otros que omito por no alargar el texto) nos dice que debemos igualar a cero la derivada primera de una variable con respecto a la otra. Es verdad que para ello la relación entre ambas variables no ha de ser lineal porque no obtendríamos de ello ningún resultado significativo. Esta es la razón por la que no podemos utilizar este método para calcular la tasa máxima de ganancia del sistema. Sraffa considera que esta tasa máxima se puede escribir como la ecuación (2), pero sustituyendo directamente su tasa máxima $\boldsymbol{g}_{\mathrm{w}=\mathrm{o}}$ por la razón-patrón $\boldsymbol{R}$. Por definición esta razón-patrón surge de la construcción de la mercancía-patrón a partir de los datos de la realidad, es decir, a partir de los productos finales $Y$ y de los medios $X$ :

$$
Y Q I=(1+R) X Q I
$$

siendo $\mathbf{Q}$ una matriz (en este caso diagonal) de multiplicadores e I el vector vertical de unos $^{15}$. Lo que se ha hecho en realidad es derivar los productos finales $Y$ en otros productos $Y Q I$ y los medios $X$ en $X Q I$ de tal forma que los excedentes relativos de cada una de las mercancías -que son distintos en el mundo real-, sean iguales en el mundo de la mercancía-patrón. Llamamos excedentes relativos al cociente:

$$
e_{i}=\left(\sum_{j=1}^{n} y_{i j}-\sum_{j=1}^{n} x_{i j}\right) / \sum_{j=1}^{n} x_{i j} \quad \forall i=1 \text { a } n
$$

La construcción de la mercancía-patrón viene derivado de que, al aplicar Perron-Frobenius en (13), obtenemos que todos los excedentes relativos son iguales. A este excedente especial le llamamos -porque así lo hace Sraffarazón-patrón $\boldsymbol{R}$. Es decir, que en (13) se cumple que:

$$
e_{1}=e_{2}=\Lambda \Lambda=e_{n}=R
$$

\footnotetext{
${ }^{15}$ En el aspecto formal de la obra de Sraffa, los multiplicadores son los elementos de un vector vertical. Aquí, en cambio, hemos ampliado el concepto de multiplicador a una matriz porque ello nos permite la posibilidad de que algunos multiplicadores de la matriz sean menores que uno, pero con tal de que la suma horizontal de los elementos de la matriz sea mayor (o igual) que cero. Dicho de otra forma, si llamamos $\boldsymbol{Q}_{\mathrm{s}}$ el vector de multiplicadores de Sraffa y lo que aquí entendemos por matriz de multiplicadores general (que llamaremos $\boldsymbol{Q}_{g}$ ), tenemos que la relación entre vector de Sraffa y multiplicadores generalizados es: $\mathbf{Q}_{\mathrm{s}}=\mathbf{Q}_{\mathrm{g}} \mathbf{I}$, siendo I el vector vertical de unos. Ninguno de los aspectos económicos considerados cambia por ello. La condición comentada (restricción) es la de que $\boldsymbol{Q}_{\mathrm{g}} \mathbf{l} \mathbf{0}$. (igual a cero si, por analogía con la aplicación de Perron-Frobenius, aceptamos esta condición como equivalente a la versión débil del teorema).
} 
La ecuación (13) puede ser escrita como:

$$
\frac{1}{1+R} \times(Q I)=(1+R) Y^{-1} X(Q I)
$$

tras llevar al escalar $(\mathbf{1}+\boldsymbol{R})$ al primer término y pre-multiplicar por la inversa de $\boldsymbol{Y}$ la ecuación (13). En la producción simple, $Y$ es una matriz diagonal, por lo que el producto $Y^{-1} X$ es positivo dado que no tendría sentido que ningún producto final de $\boldsymbol{Y}$ ni ningún medio de $\boldsymbol{X}$ fueran negativos. Tanto $\boldsymbol{Y}$ como $\boldsymbol{X}$ son cuadradas. Sólo falta considerar al producto $\boldsymbol{Y}^{-1} \boldsymbol{X}$ como irreducible para aplicar la versión fuerte del teorema de Perron-Frobenius. Con ello obtenemos que $\mathbf{1} /(\mathbf{1}+\boldsymbol{R})$ va a ser el autovalor más alto de todos los autovalores tomados en términos absolutos; además que el vector $Q \mathbf{l}$ va a ser su autovector correspondiente por la derecha, con la propiedad de que todos sus elementos van a ser positivos ${ }^{16}$. Vayamos ahora al sistema original de ecuaciones dado por (1) y pos-multipliquemos por el vector QI, cuyos elementos ya los hemos obtenido a partir de (16) y que dependen sólo de las variables físicas del sistema, es decir, de $\boldsymbol{Y}$ y $\boldsymbol{X}$, y no de las variables monetarias (gracias a PerronFrobenius). Se obtiene entonces:

$$
P Y Q I=(1+g) P X Q I+w L Q I
$$

Si ahora pre-multiplicamos la ecuación (13) por el vector de precios $\boldsymbol{P}$ sale:

$$
P Y Q I=(1+R) P X Q I
$$

Las ecuaciones (17) y (18) corresponden a un sistema económico de mercancía-patrón análogo al sistema de economía real dado por las ecuaciones (1), (2) y (3), pero con dos diferencias: 1) aquí el sistema económico se ha transformado mediante $Q \mathbf{I}$ en otro sistema (posiblemente reducido) tal que todos los excedentes relativos de todas los bienes y servicios (mercancías en Sraffa) son iguales; 2) que en lugar de tener una tasa máxima de ganancia $\boldsymbol{g}_{\mathrm{w}=0}$ que obtiene Sraffa (aunque él lo llama directamente razónpatrón, es decir, el $\boldsymbol{R}$ de aquí), tenemos la razón-patrón producto de la definición de excedente relativo y de la aplicación del teorema de PerronFrobenius al sistema así definido (ecuación (13)). Vamos a añadir a las ecuaciones (17) y (18) un numerario análogo al definido por la ecuación (3).

$$
P Y Q I-P X Q I=1
$$

\footnotetext{
${ }^{16}$ Además un teorema nos asegura que el autovalor del producto $\mathbf{Y}^{-1} \mathbf{X}$ va a ser el mismo que el autovalor $\mathbf{X Y}^{-1}$ (no así los auvectores correspondientes).
} 
Pues bien, del conjunto de ecuaciones (17), (18) y (19) sale:

$$
g=(1-w L Q I) R
$$

Vemos en este apéndice cómo la simplificación llevada por Sraffa obliga a hacer dos consideraciones: 1) que la tasa máxima de ganancia derivada de hacer cero la tasa de ganancia en la ecuación (1) de definición del sistema de precios es equivalente (igual) al resultado de calcular la razón-patrón una vez definido la mercancía-patrón (ecuación (13)); 2) que al tomar dos numerarios (cosa que se puede hacer) supone igualar a 1 las ecuaciones (3) y (4) del sistema (cosa que no se puede hacer). Por estos motivos, la ecuación correcta de distribución de la renta 0 , dicho con más propiedad, del reparto del excedente, sería la (20) y no la que propone Sraffa de $\boldsymbol{g}=(\mathbf{1}-\boldsymbol{w}) \boldsymbol{R}$. Sin embargo todo el desarrollo posterior y la utilización de la famosa ecuación de Sraffa no cambian las conclusiones económicas allí donde se emplea (por ejemplo, en la reducción del capital a trabajo fechado o en el desplazamiento de los métodos de producción). No obstante, no se trata sólo de diferencias formales, sino de que detrás de éstas están las diferencias conceptuales que hemos tratado de puntualizar.

Una tercera consideración viene a cuento de por qué hemos llamado a la tasa máxima de ganancia de Sraffa $\boldsymbol{g}_{\mathrm{w}=\mathbf{0}}$. En el texto del artículo hemos deducido con la ayuda de la tasa máxima de salario (que Sraffa no considera formalmente, es decir, que nunca tradujo a una ecuación) que la tasa máxima de ganancia a partir de esos supuestos (1), (2) y (3) (pero no el (4)), más el de esa tasa máxima de salarios, es decir, de que:

$$
P Y=w_{m} L+P X
$$

corresponde a la ecuación:

$$
g_{m}=\frac{L X^{-1}[Y-X] I}{L I}
$$

Tasa máxima distinta de la que aparece como variable independiente en (12) $\boldsymbol{g}=(\mathbf{1}-\boldsymbol{w} \boldsymbol{L} \mathrm{I}) \boldsymbol{g}_{\mathrm{w}=\mathbf{0}}$. La razón de esta diferencia es que, para llegar a la tasa máxima de ganancia $\boldsymbol{g}_{\mathrm{m}}$ de (22) hemos añadido una ecuación más al sistema que es la 
derivada de hacer cero la tasa de ganancia ${ }^{17}$. Pero, dado que Sraffa considera que la tasa máxima de (12) es la razón-patrón $\boldsymbol{R}$, vamos a comparar la ecuación (22) con una formulación en la que aparezca esta razón-patrón. Lo mejor para ello es partir de la propia definición de razón-patrón, que es la ecuación $Y Q I=(\mathbf{1}+R) X Q I$. Si ahora pre-multiplicamos esta última ecuación por el vector de inputs de trabajado $L$ obtenemos:

$$
L Y Q I=(1+R) L X Q I
$$

Y despejado $\boldsymbol{R}$ en la anterior sale:

$$
R=\frac{L[Y-X] Q I}{L X Q I}
$$

Vemos ahora la diferencia entre las tasa máximas de ganancia $\boldsymbol{g}_{\mathrm{m}}, \boldsymbol{g}_{\mathrm{w}=0}$ y $\boldsymbol{R}$. Y eso que no hemos considerado de la que habla Sraffa de la menor de las razones-patrón, que ya hemos discutido en el texto principal. Tenemos, ya no 3 tasas máximas, sino 4 , pero se mantiene el criterio de que de Sraffa sólo se deducen 3 porque identificó (aunque sin justificarlo) la tasa $\boldsymbol{g}_{\mathrm{w}=0}$ (derivada de hacer cero lo salarios en la ecuación que definía su sistema) con la tasa máxima que surge de la mercancía-patrón (que es la razón-patrón $\boldsymbol{R}$ ). Hay que decir que para que fueran iguales la tasa máxima $\boldsymbol{g}_{\mathrm{m}}$ de (22) y la razón-patrón $\boldsymbol{R}$ de (24) debería cumplirse que:

$$
\frac{L Y Q I}{L X Q I}=\frac{L X^{-1} Y I}{L I}
$$

lo cual sólo puede ser cierto por casualidad dado que el vector de multiplicadores $Q \mathbf{I}$ se ha obtenido merced a consideraciones de mercancíapatrón y la utilización del teorema de Perron-Frobenius. No obstante, la ecuación (25) abre nuevas posibilidades de de cálculo de una mercancíapatrón distinta de la procedente del teorema. Mediante la programación lineal puede calcularse el vector resultante QI de multiplicadores con una función objetivo como la (25), tomando como variables los $\boldsymbol{n}$ QI multiplicadores y con las restricciones de que $L X Q \mathbf{I}=L I=1$. A éstas se les puede añadir la condición de que $\mathbf{Q}=\mathbf{1}$ y de que $\mathbf{Q}>\mathbf{1}$. De no cumplirse esta última condición y permitir que algunos multiplicadores $\boldsymbol{q}_{\mathrm{ij}}$ fueran cero, podría ocurrir que no existiera un máximo si la derivada de un multiplicador respecto a otro $\left(\mathrm{dq}_{\mathrm{ij}} / \mathrm{d} \mathbf{q}_{\mathrm{k}}\right.$, variando

\footnotetext{
${ }^{17}$ Un resultado interesante es el resultado de igual las tasas máximas $\boldsymbol{g}_{\boldsymbol{w}=0}$ derivada de la ecuación $\mathbf{g}=(\mathbf{1}-\mathbf{w L I}) \boldsymbol{g}_{\mathrm{w}=0}$ y la tasa máxima $\boldsymbol{g}_{\mathrm{m}}$ de $\boldsymbol{g}_{\mathrm{m}}=\mathbf{L} \mathbf{X}^{-1}(\mathbf{Y}-\mathbf{X}) \mathbf{I} / \mathbf{L I}$. El resultado es que se cumple: $\mathbf{g}=\left(\mathbf{w}_{\mathrm{m}}-\mathbf{w}\right) \mathbf{L} \mathbf{X}^{-1}(\mathbf{Y}-\mathbf{X}) \mathbf{l}$. Hay que tener en cuenta para llegar a este resultado hemos utilizado que la tasa máxima de salario $\mathbf{w}_{\mathrm{m}}$ es igual a $\mathbf{1 / L I}$.
} 
$\boldsymbol{i}, \boldsymbol{j}, \boldsymbol{k}, \boldsymbol{I}$ de $\mathbf{1}$ a $\boldsymbol{n}$ ) de la función objetivo tuviera el mismo signo que la misma derivada en las restricciones.

Hemos comprobado a lo largo del texto del artículo y de los tres apéndices cómo la consideración de la tasa máxima de ganancia que hace Sraffa ofrece más enjundia y dificultades de las que una primera e ingenua lectura de los primeros capítulos de Producción de mercancías por medio de mercancías pudiera derivarse.

\section{Bibliografía}

Dobb, M.: "The Sraffa system and the critique of neoclassical theory of distribution", 1970.

Kurz y Salvadori: "Sraffa and the mathematicians: Frank Ramsey and Alister Watson", en "Piero Sraffa's Political Economy, edit Routledge,

Kurz D. Heinz; "Critical Essays on Piero Sraffa's Legacy in Economics”, 2000, Cambrigde University Press.

Mora Plaza, A.; "Descifrando a Sraffa”, Editorial Académica Española, 2011.

Murga, Gustavo: "Piero Sraffa".

http://marxismo.cl/portal/index.php?option=com_content\&task=view\&id=100\&lte $\operatorname{mid}=1$

Neri, Salvador: "Besicovitch, Sraffa and the existence of Standard Commodity", 2010:

http://host.uniroma3.it/eventi/sraffaconference2010/abstracts/pp_salvadori.pdf

Pasinetti. L.: "Critical of the neoclassical theory of growth and distribution". Está en la red:

http://www.unicatt.it/docenti/pasinetti/pdf_files/Treccani.pdf 
Pasinetti, L.: "Structural Change and Economic Growth: a theoretical essay on the dynamics of Wealth of Nations", 1981, Cambridge University Press.

Pasinetti, L.: "Rate of profit and income distribution in relation to the rate of economic growth", 1961/2.

Pasinetti, L.: "Switches of technique and the rate of return in Capital Theory", 1969.

Pasinetti, L.: "Lecciones de teoría de la producción" ("Lezioni di teoria della produzioni", 1975), 1983, FCE.

Potier, J.P.: "Piero Sraffa", 1994, edicions Alfons Magnànim.

Roncaglia, Alessandro: "Piero Sraffa", Edit. Palgrave MacMillan, 2009.

Subiza Martínez, B.: "Juegos matriciales y su aplicación a la teoría PerronFrobenius", U. de Alicante; http://www.ine.es/revistas/estaespa/112_3.pdf

Solow, R.: "The interest rate and transition between techniques", 1967.

Sraffa, Piero: "Produción de mercancías por medio de mercancías" (Production of commodities by means of commodities, 1960), 1975, Oikos-Tau.

Vegara, J. M.: “Economía política y modelos multisectoriales”,1979, edit. Tecnos. 